\title{
Structural changes in the outer retinal layers following blunt mechanical non-perforating trauma to the globe: an experimental study
}

\author{
R. BLIGHT AND J. C. DEAN HART \\ From the Departments of Pathology and Ophthalmology, University of Bristol
}

SUMMARY Structural changes in the external retinal layers of pigs' eyes that had previously been subjected to blunt non-perforating mechanical injuries have been described. Within minutes of trauma fragmentation of the photoreceptor outer segments and damage to the retinal pigment epithelium was recorded in areas of retina adjacent to the missile impact site on the sclera. One week after injury the disrupted photoreceptor outer segments disappeared, and the inner segments came to lie adjacent to the retinal pigment epithelium. Evidence of regeneration of photoreceptor outer segments was noted 2 weeks later.

Disturbances of the retinal pigment epithelium are common sequelae to blunt ocular injuries in man, both at the posterior pole (Haab, 1888) and in the retinal periphery (Davidson, 1936). Few reports have appeared describing damage to the photoreceptors, though involvement of these cells might be expected owing to the intimate anatomical and biochemical relationship between the photoreceptor outer segments and the retinal pigment epithelium.

Absence of photoreceptors was recorded in a patient with a traumatic retinal detachment after a concussional ocular injury (Tillema, 1937), and more recently selective loss of these elements was reported in a patient who had recently sustained a blunt perforating ocular injury but did not develop a retinal detachment (Cogan, 1969). Disintegration of the external retina, with formation of vacuole-like cavities in the photoreceptor outer segment layer, was observed in the eyes of experimental animals (rabbits) examined less than 2 hours after being subjected to blunt non-perforating mechanical trauma (Petropavlovskaya and Bogoslovsky, 1966).

Damage to the outer retinal layers following blunt non-perforating mechanical injuries has been attributed to concussional forces: (1) Producing spasm of the choroidal circulation (Bonnet and Sedan, 1954) leading to ischaemic tissue necrosis; (2) acting on the retinal epithelial cells to induce these to become mobile phagocytes, which digest the

Address for reprints: J. C. Dean Hart, FRCS, Bristol Eye Hospital, Lower Maudlin Street, Bristol BS1 2LX adjacent layers of the retina (Cogan, 1969); (3) by disrupting the pigment epithelium, causing lysosomal liberation with the same end result as above (Cogan, 1969).

Since opportunities to examine microscopically any cellular changes which occur after concussional injuries to the human retina rarely arise, especially within a few days of the event, the eyes of experimental animals (pigs) were subjected to blunt traumatic injury. At predetermined times the globes were removed and examined histologically.

\section{Materials and methods}

EXPERIMENTAL ANIMALS

All trauma experiments were performed on $20-\mathrm{kg}$ store pigs. The pig was selected because this animal has a holangiotic retinal blood supply with a capillary meshwork of about the same size, and supplying identical retinal layers, as the human (Rootman, 1971). The eyeball dimensions are similar to those of man, thus the forces liberated by a missile impacting against the globe might be expected to act on the intraocular tissues in a somewhat similar way.

\section{TRAUMA}

This was produced by firing a pellet from a modified airgun to give a missile impact energy of approximately $0.56 \mathrm{ft} \mathrm{lb}(0.08 \mathrm{~kg} \mathrm{~m})$. At this energy level ocular perforation did not occur (Weidenthal and Schepens, 1966). The slug was made to impact perpendicularly against the sclera in the 3 o'clock 
position in the right eye and in the 9 o'clock position in the left eye, 4 to $5 \mathrm{~mm}$ from the corneoscleral junction.

\section{ANAESTHESIA}

All experiments involving ocular trauma were performed under general anaesthesia. This was induced and maintained by the animal breathing a mixture of fluorothane in nitrous oxide and oxygen on open circuit, delivered through a flexible rubber nose cone which fitted over the animal's snout. No additional premedication was used.

\section{LIGHT AND ELECTRON MICROSCOPY}

Eyes were carefully but quickly enucleated prior to killing the animal. Seven animals formed the basis of this investigation and the globes were removed at the following time intervals after trauma: within 1 minute, at 1 day, 7 days, 3 weeks, and 2 months.

After removal of the eye a wide incision was made through the ocular coats in the region of the pars plana with a dermatome blade, and the globe was immediately placed in $150 \mathrm{ml}$ of solution at $4^{\circ} \mathrm{C}$ containing $4 \%$ glutaraldehyde buffered with $0.6 \mathrm{M}$ sodium cacodylate to give a $\mathrm{pH}$ of $7 \cdot 4$. After 15 minutes' initial fixation the incision was completed and the posterior eye cup returned to the fixative. Two hours later the posterior cup was transferred to cold $0.5 \mathrm{M}$ sucrose solution buffered with $0.2 \mathrm{M}$ sodium cacodylate, and $5 \mathrm{~mm}$ discs of full-thickness retina, choroid, and sclera were trephined out of selected areas of the retina. These included samples from the mid-nasal and mid-temporal regions and were taken along a horizontal plane which passed through the point of impaction of the missile.

Tissue was post-fixed in Millonig's phosphate buffered osmium tetroxide for $1 \frac{1}{2}$ hours. After dehydration in ascending concentrations of ethanol the samples were embedded in Spurr resin and sections cut on a Reichert OMU 2 ultramicrotome. Thick sections at $0.5 \mu \mathrm{m}$ were stained with toluidine blue for photomicrography with Nomarski differential interference contrast. Thin sections were cut, mounted on copper grids, stained with uranyl acetate and lead citrate, and examined with the Phillips EM300 electron microscope.

\section{Results}

\section{Changes in the outer retinal layers of eyes}

removed within 1 minute of trauma

An interference contrast photomicrograph shows the normal appearance of a pig's external retinal layers (Fig. 1). Immediately after trauma fragmentation of the photoreceptor outer segments occurred in the nasal retina adjacent to the site of missile impact on the outer coats of the globe, but no loss of continuity of the retinal pigment epithelial layer or ruptures of Bruch's membrane developed. Occasional haemorrhages were present in the outer retinal layers, but the choroidal vessels appeared to be everywhere intact (Fig. 2). When compared with its appearance in Fig. 1, the outer limiting membrane showed no evidence of dehiscences or breaks.

Electron micrographs of the nasal retina also show that marked disruption of the photoreceptor outer segments had occurred. The fragments consisted of irregular-sized pieces recognisable as outer segment material containing 20 or more saccules and as loose skeins of disc plates. The photoreceptor inner segments appeared to be somewhat separated from each other, but the severity of damage to these structures was less marked than in the outer segments, and only a few of the cell boundaries were ruptured. Cleavage of the outer from the inner segments of the photoreceptors had occurred mainly at the level of the connecting cilia (Fig. 3). The tips of the outer segments of the photoreceptors had, with few exceptions, lost contact with the retinal pigment epithelium. Only the occasional apical process could be identified, and there was evidence of some ruptures in the retinal pigment epithelial inner cell membranes, as pigment cell particles could be observed in the area occupied by the fragmented outer photoreceptor segments. However, the outer and lateral cell boundaries, including the junctional complexes of the retinal pigment epithelial layer, appeared to be intact. Intracellular oedema of the retinal pigment epithelial cells was an obvious feature, and numerous microcystic spaces were present. Phagosomes were detected at this stage after trauma. Bruch's membrane and the choroidal vessels showed no evidence of damage, either immediately after injury or at later stages (Fig. 4).

Electron micrographs of sections obtained from the temporal retina on the opposite side of the globe to where the airgun pellet had struck the eye reveal no evidence of structural damage in the outer retinal layers (Fig. 5).

Microscopical appearances of the outer retinal layers of eyes removed 24 hours after trauma

Electron micrographs obtained from sections of the nasal retina showed that the fragments of lamellar disc material were less electron-dense than 24 hours previously, and the discrete fragments containing saccules had almost disappeared. Retinal pigment epithelial cells showed more evident intracellular oedema as the inner cell boundaries had become ballooned out. Few apical processes could be detected, and no phagosomes were observed. Isolated wandering macrophages containing electron-dense 


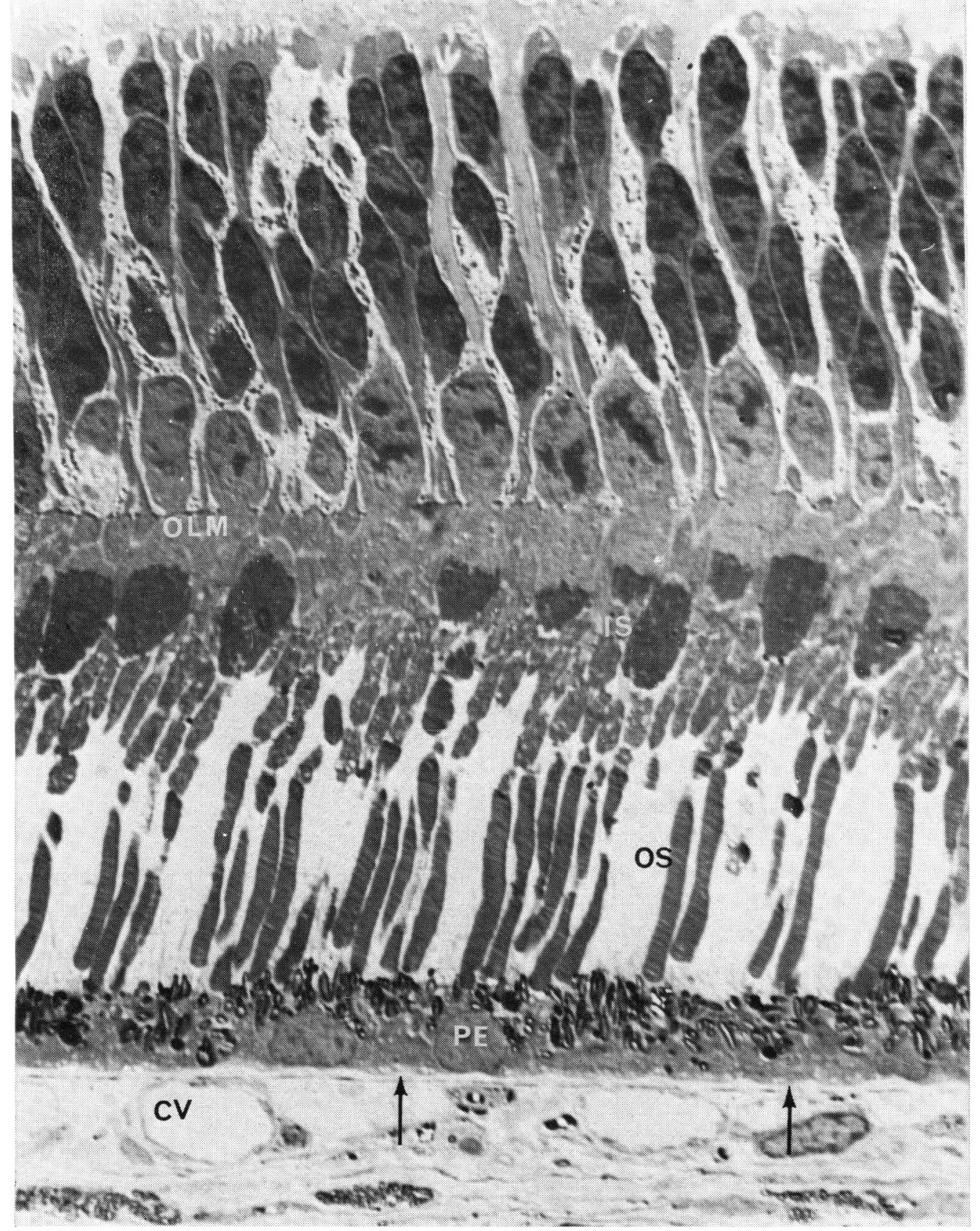

Fig. 1 Interference contrast micrograph of untraumatised retina. Outer limiting membrane, OLM: photoreceptor inner segments, IS: pigment epithelium, PE: Bruch's membrane (arrows): choroidal vessels, CV. $\times 1640$ 


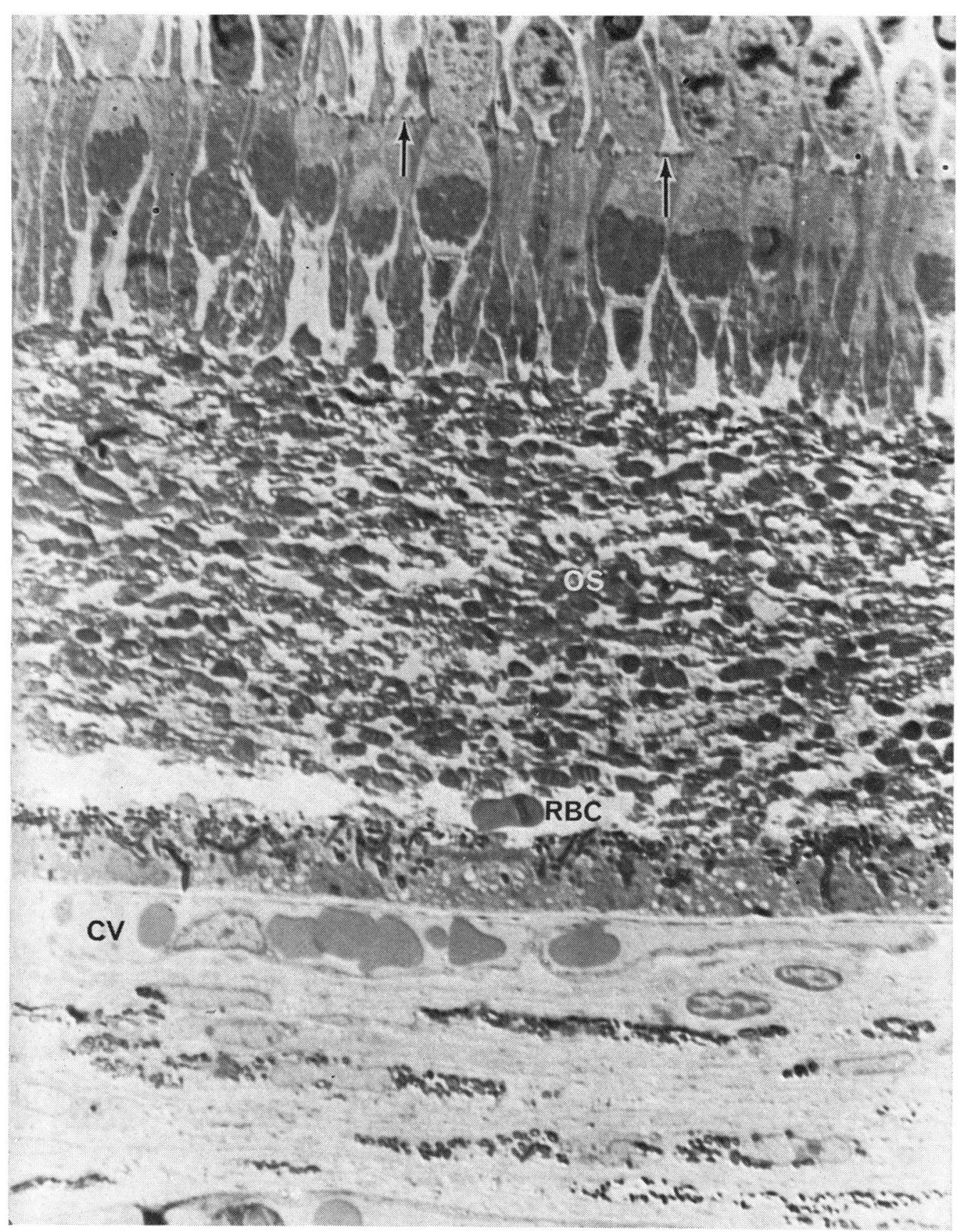

Fig. 2 Interference contrast micrograph of nasal retina of globe removed within 1 minute of trauma, showing disruption of the outer segment layer, OS. No breaks are present in the outer limiting membrane (arrows) or the pigment epithelium. Occasional haemorrhages, RBC, can be identified, but the choroidal vessels, $C V$, appear intact. $\times 1640$ 


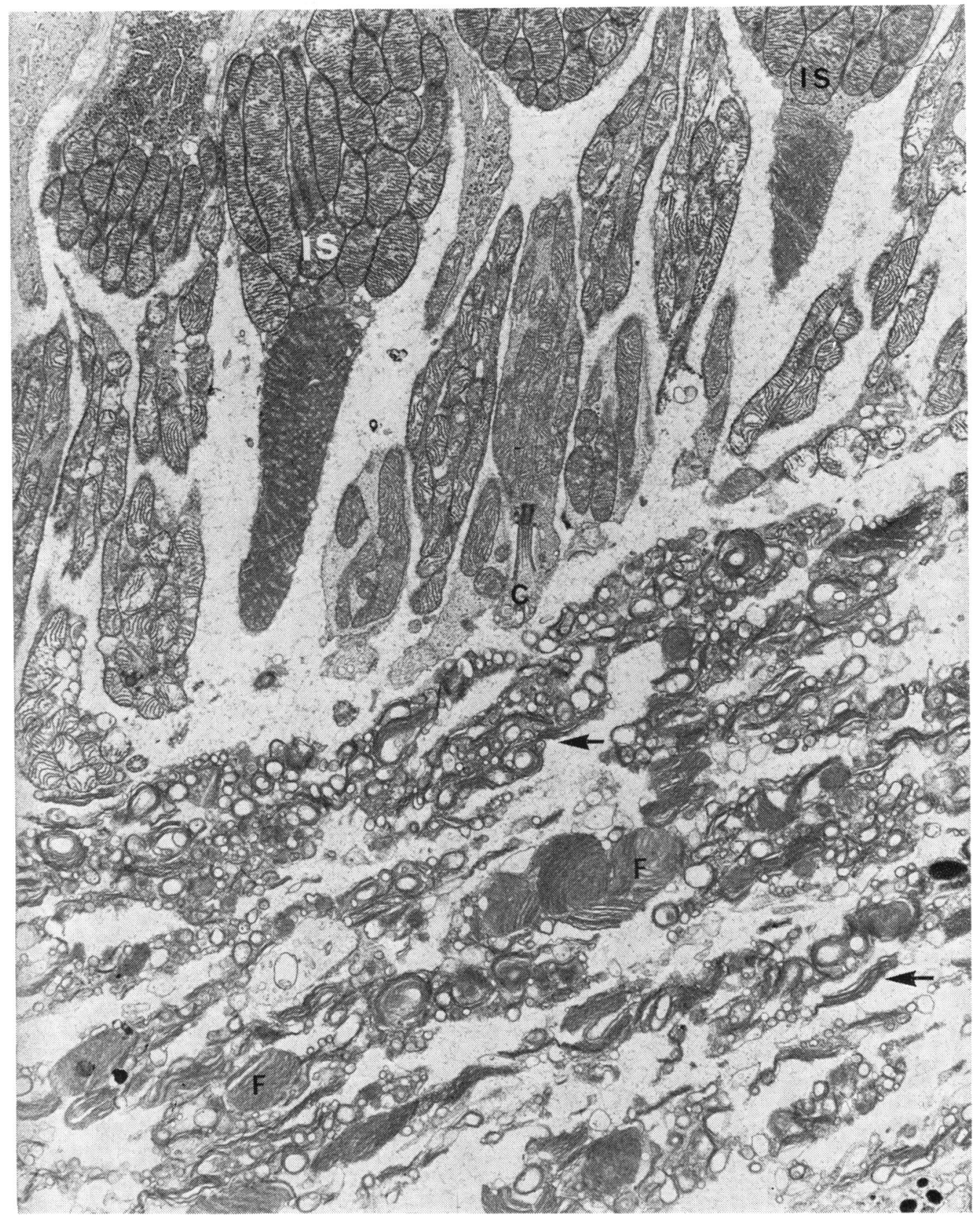

Fig. 3 Electron micrograph of nasal retina of globe removed within 1 minute of trauma, showing fragmented outer segment material consisting of loose discs (arrows), and fragments containing saccules, $F$. Inner segments, IS, are separated from each other, but show less damage than outer segments, which have split off at the level of the connecting cilia, C. $\times 6900$ 


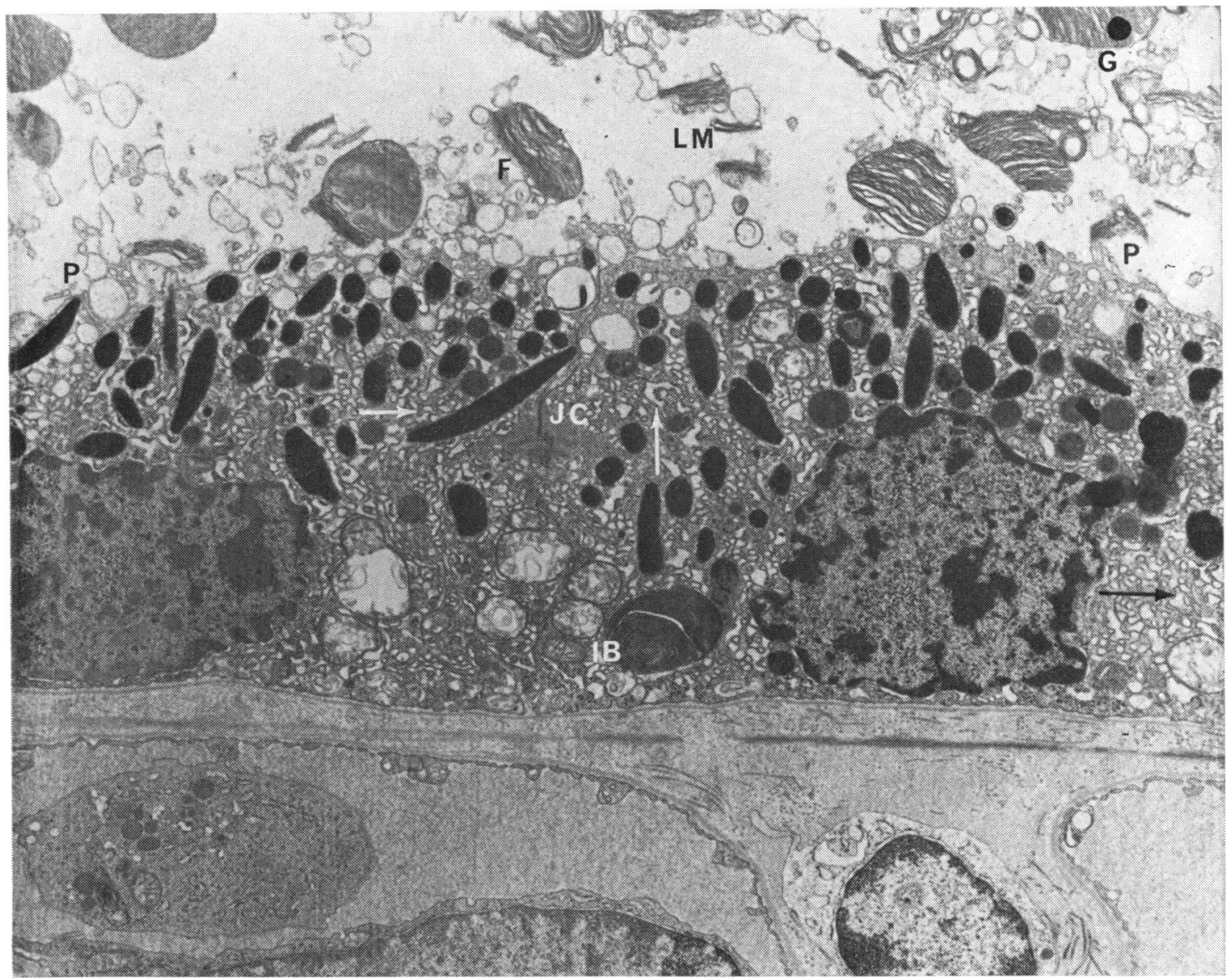

Fig. 4 Electron micrograph of nasal retina of globe removed within 1 minute of trauma. There are numerous microcystic spaces in the cytoplasm of the pigment epithelium (arrows). A few stunted apical processes, $P$, can be seen, and some inner cell membranes have ruptured with dispersion of pigment granules, $G$. The lateral and outer cell boundaries are intact, including the junctional complexes, JC. Lamellar inclusion bodies, IB, are present. Fragmented outer segments consist of loose lamellar material, LM, and fragments containing saccules, $F . \times 8680$

material derived from the pigment epithelium were seen only in the occasional section (Fig. 6).

Sections of the outer retinal layers obtained from the temporal retina failed to demonstrate any abnormal features.

\section{Microscopical appearances of the outer retinal layers of eyes removed 7 days after trauma}

Interference contrast micrographs of the nasal retina show that the photoreceptor inner segments had become approximated to the inner cell boundaries of the pigment epithelium. The space which contained the fragmented photoreceptors had disappeared (Fig. 7).

Electron micrographs confirm that the layer previously occupied by the photoreceptor outer segments had collapsed and the cell debris dispersed. Only a few stumps of outer segment material were identifiable, which were possibly regenerating photoreceptor outer segments (see below). The retinal pigment epithelium appeared to be less swollen, and apical processes were again observed in large numbers (Fig. 8). No phagocytic activity could be detected by the presence of wandering macrophages, and no phagosomes were seen within the pigment epithelial cells. Sections obtained from the temporal retina appeared free of structural changes.

Microscopical appearances of the outer retinal layers of eyes removed 3 weeks after trauma Interference contrast micrographs of the nasal retina show that the outer segments had reappeared (Fig. 9). 


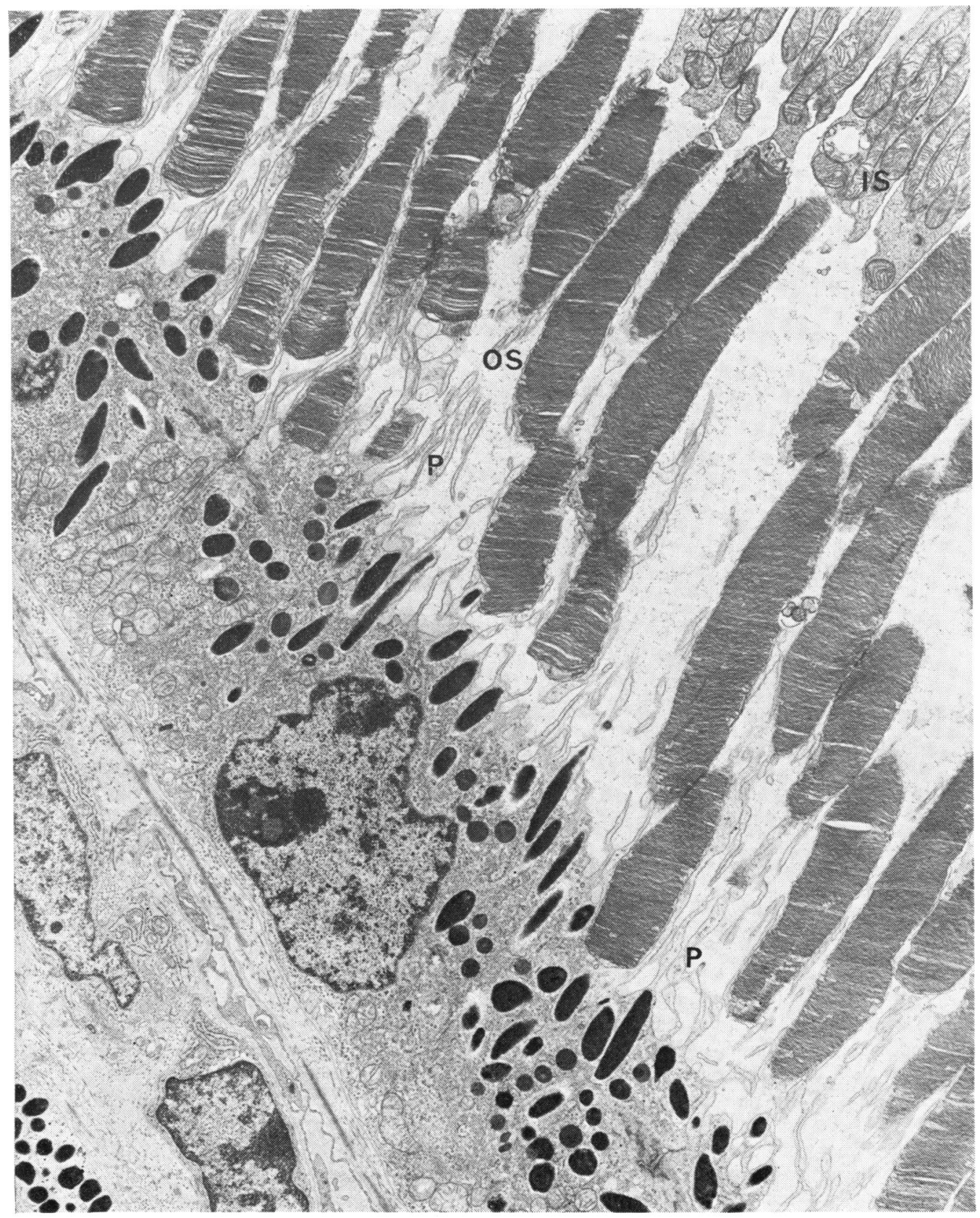

Fig. 5 Electron micrograph of temporal retina of globe removed within 1 minute of trauma, showing pigment epithelium with numerous apical processes, $P$, and normal appearances of photoreceptor inner, IS, and outer segments, OS. $\times 6900$ 


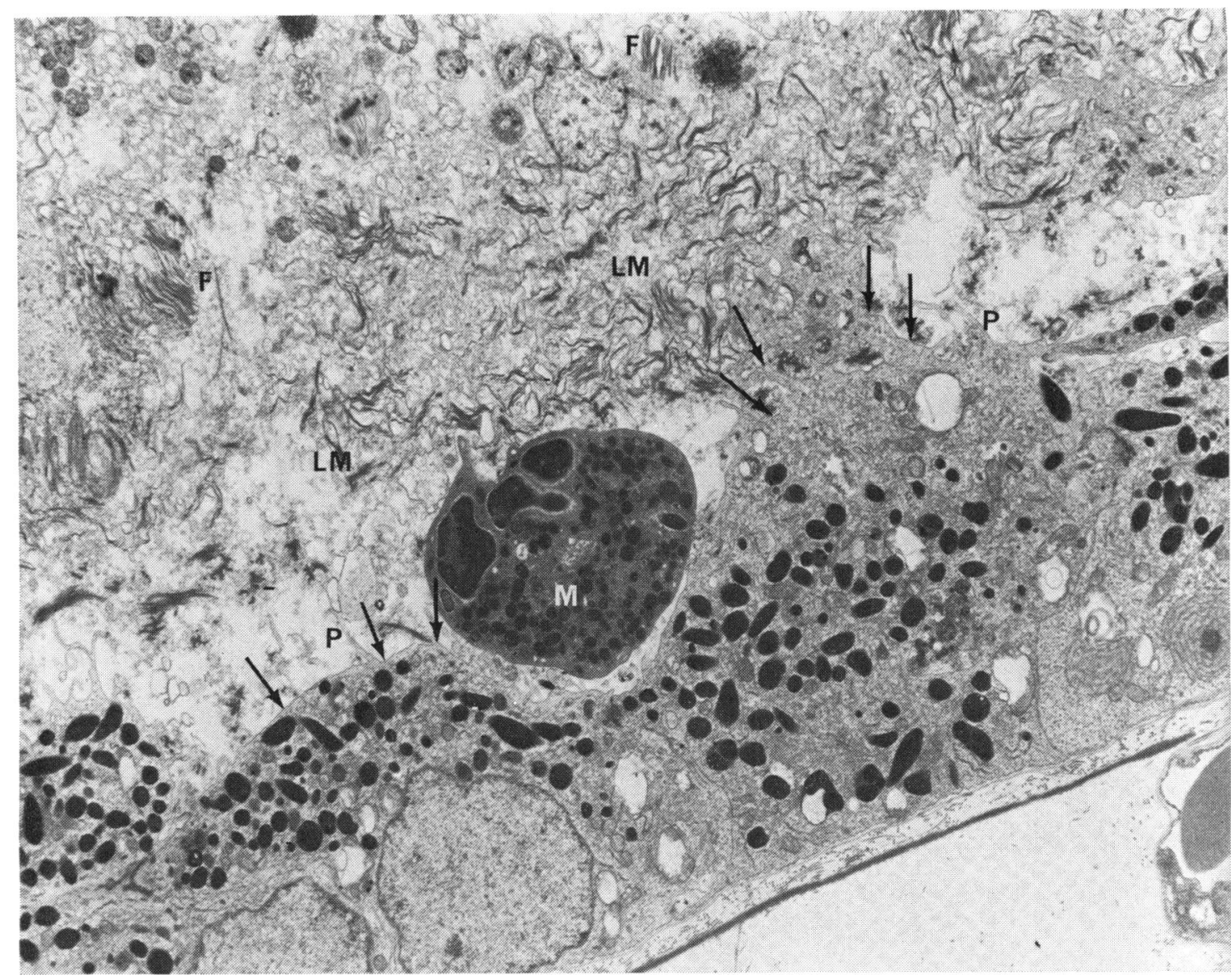

Fig. 6 Electron micrograph of nasal retina of eye removed 1 day after trauma. There is ballooning of the pigment epithelium inner cell membrane (arrows), and few apical processes, $P$, can be identified. Loose disc material, LM, is less electron-dense than 24 hours previously and few fragment containing saccules, $F$, remain. The occasional macrophage, $M$, containing pigment material is present. $\times 5960$

Electron micrographs show that a complex pattern of changes had occurred in the outer retinal zones. There was evidence of regeneration of outer segment material, some of which was fairly orderly and some extremely disorganised. Lamellar material in some outer segments had been produced in the form of whorls surrounding clear central spaces, as ribbonlike streamers and, in some cells, was present in the inner segment (Fig. 10). In other outer segments cystic spaces could be seen between the saccules, abnormal orientation of disc plates was evident in the tips of the processes, and large portions of the outer segments were filled with amorphous ground material (Fig. 11). Many saccules were stunted. Phagosomes were again noted in the pigment epithelial cells. Studies of the outer layers of the temporal retina showed no abnormal features.
Microscopical studies of outer retinal layers of eyes removed 2 months after trauma

Electron micrographs of nasal retina show that the production of outer segment material had now developed along more normal lines and that, in the majority of the outer segments, saccules were correctly orientated. The tips of the photoreceptor outer segments had established a normal relationship with the pigment epithelial cells, but in a few photoreceptors disorganised production of lamellar plate material continued (Fig. 12).

\section{Discussion}

Selective loss of photoreceptor outer segments has been reported in man following detachments secondary to malignant tumours (Kroll, 1969), and 


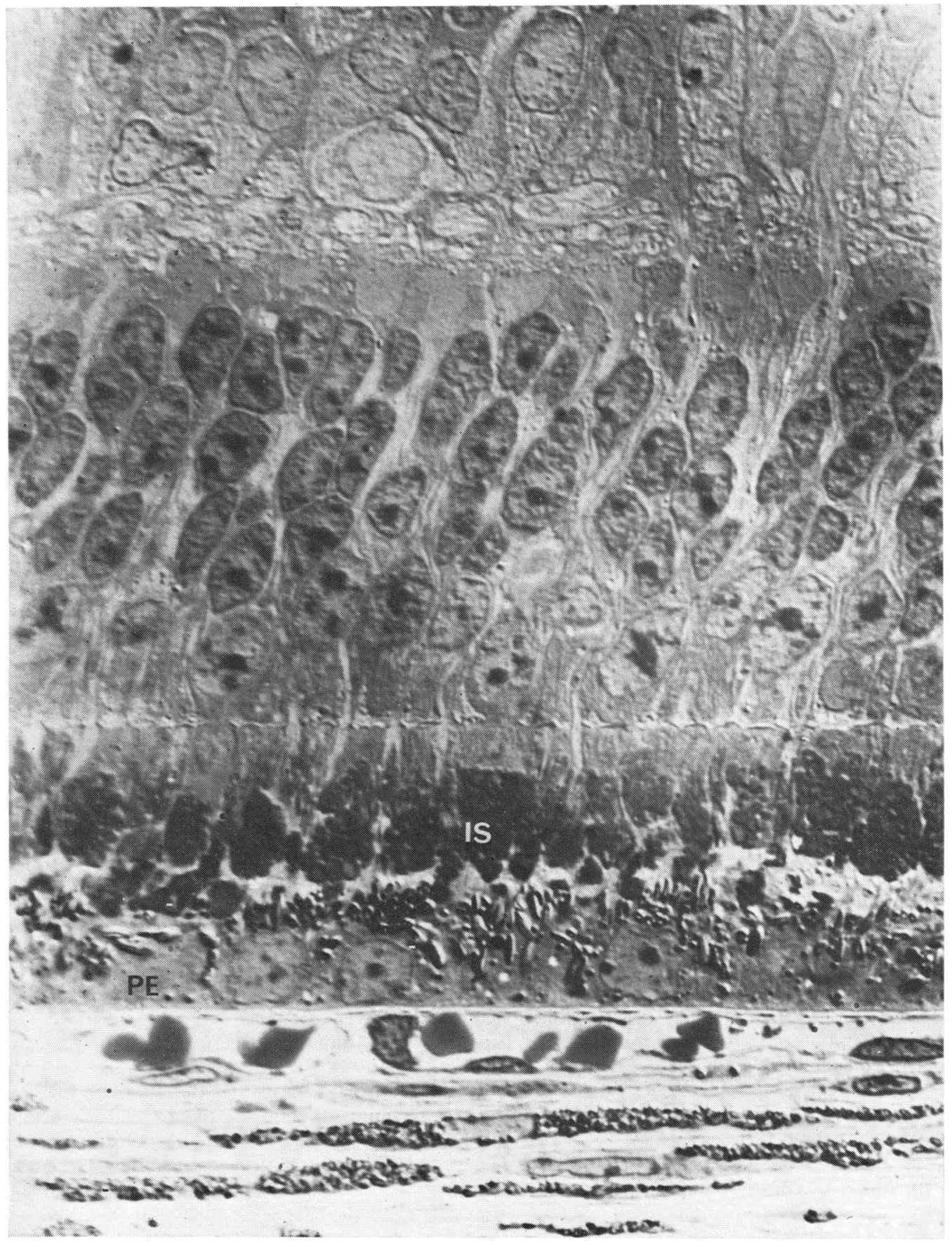

Fig. 7 Interference contrast micrograph of nasal retina of eye removed 1 week after trauma, showing approximation of inner segment, IS, to the pigment epithelium, PE. $\times 1640$ 


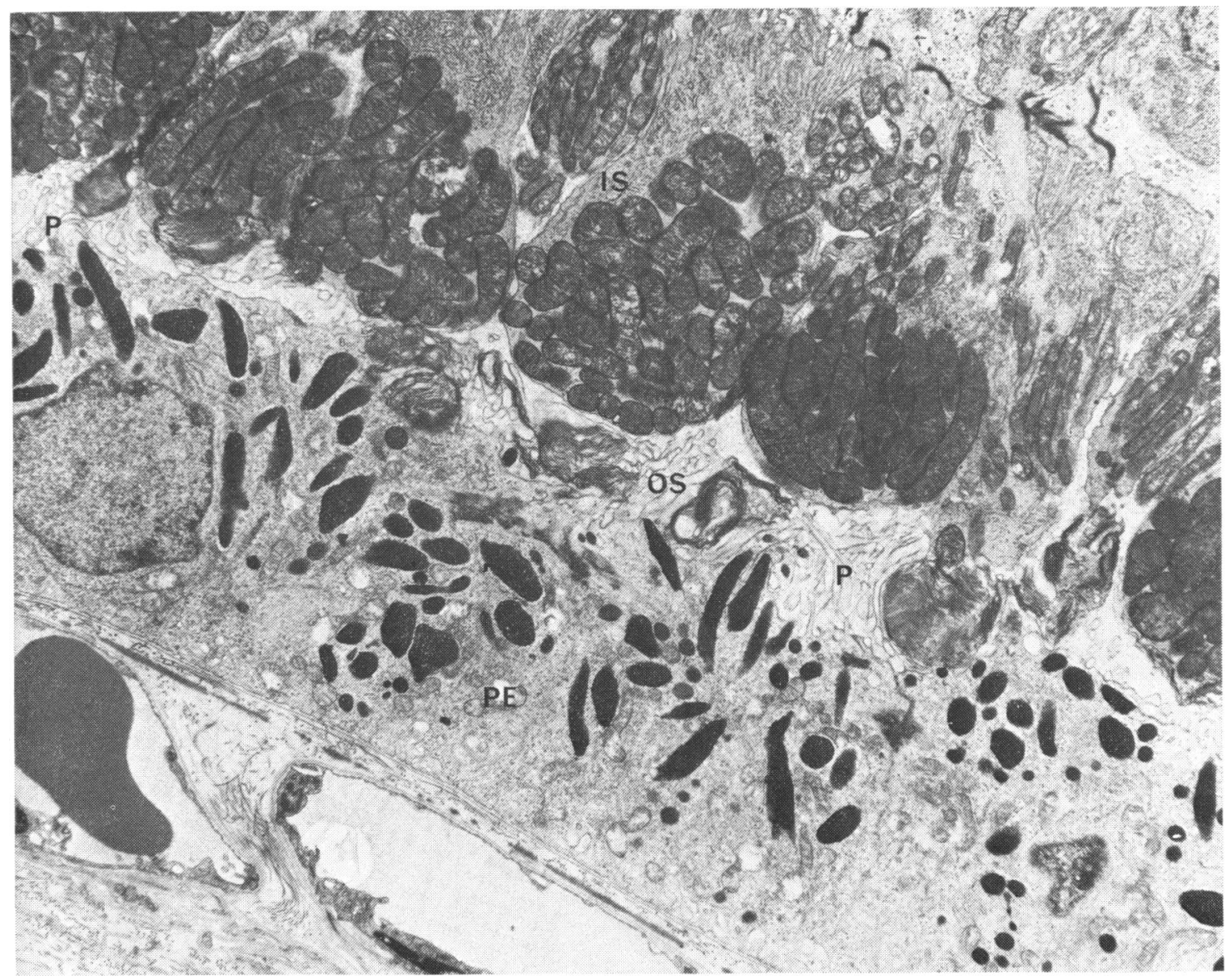

Fig. 8 Electron micrograph of nasal retina of eye removed 1 week after trauma. The zone previously containing fragmented outer segment material is almost obliterated. The inner segments, IS, lie adjacent to the pigment epithelium, PE. Only a few pieces of outer segment material, $O S$, are present. Apical processes, $P$, are seen in large numbers. $\times 5960$

studies on animals with experimental retinal detachments showed that these structures gradually atrophied (Kroll and Machemer, 1968). It is possible that the absence of photoreceptors noted in patients with traumatic detachments of the retina (Tillema, 1937; Crouch and Apple, 1974) occurred, in part, because contact between the outer segments and the retinal pigment epithelium was lost.

In this study marked outer retinal layer cellular changes were immediately apparent after trauma. They appeared too early for ischaemia, as suggested by Bonnet and Sedan (1954), or a breakdown in the biochemical pathways between the photoreceptors and retinal pigment epithelial cells to have played a major part in producing them. Examination of the choroidal vasculature did not reveal any evidence of vessel damage either immediately after trauma or for periods of up to 2 months after the injury.

We have previously described disturbances of the nerve fibre layer of the retina in experimental animals after blunt non-perforating mechanical trauma (Hart et al., 1975) that could have resulted only from forces released at the time of missile impaction acting directly on the cellular elements of the retina. The present findings of immediate fragmentation of the photoreceptor outer segments, with separation of these structures from the inner segments at the level of the connecting cilia, loss of the apical processes of the retinal pigment epithelium, and intracellular oedema of these cells after injury can only be attributed to similar causes.

No ruptures of the junctional complexes were noted on microscopy between adjacent retinal pigment epithelial cells or at the level of the outer 


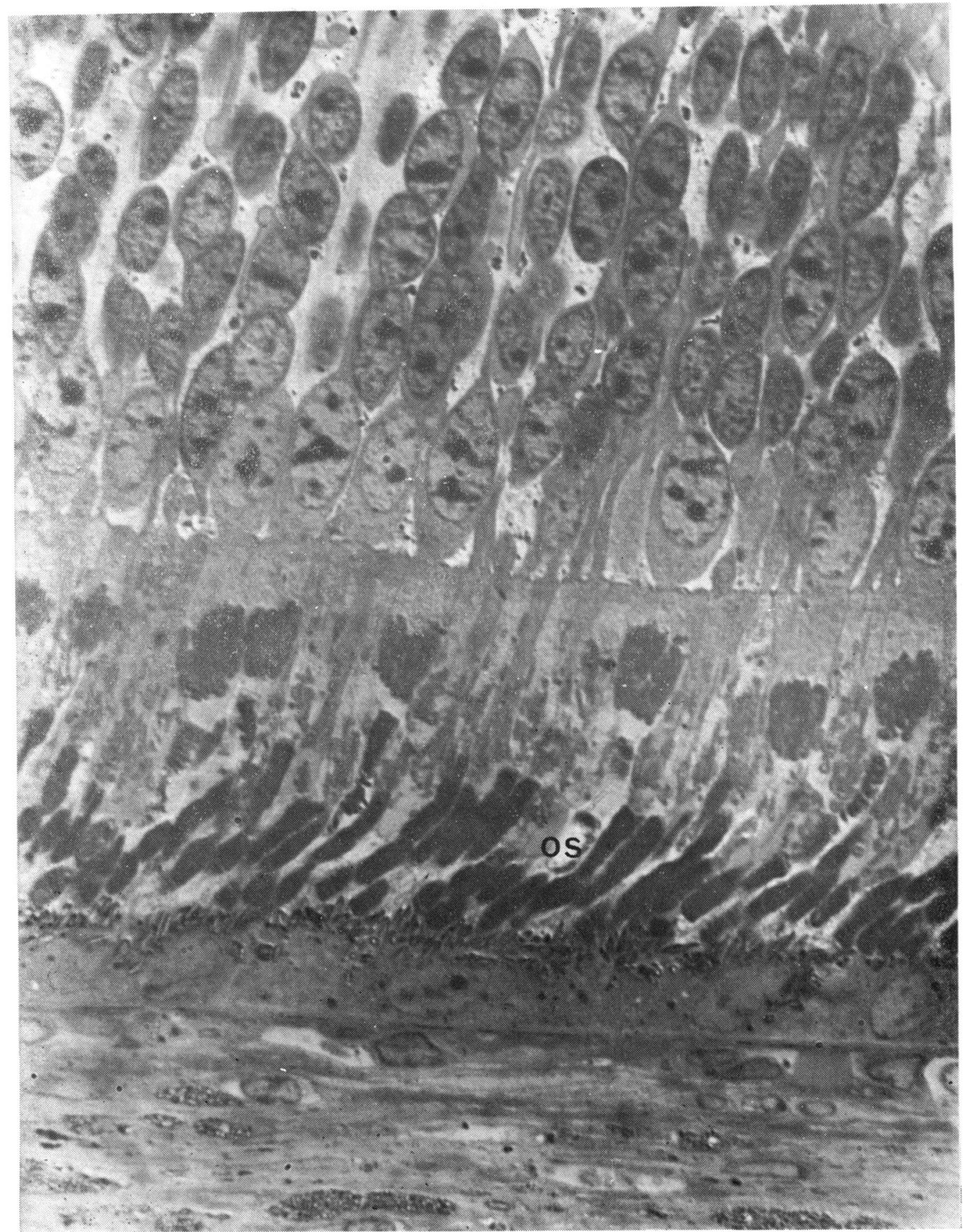

Fig. 9 Interference contrast micrograph of nasal retina of eye removed 3 weeks after trauma, showing the presence of stunted outer segments, OS. $\times 1640$ 


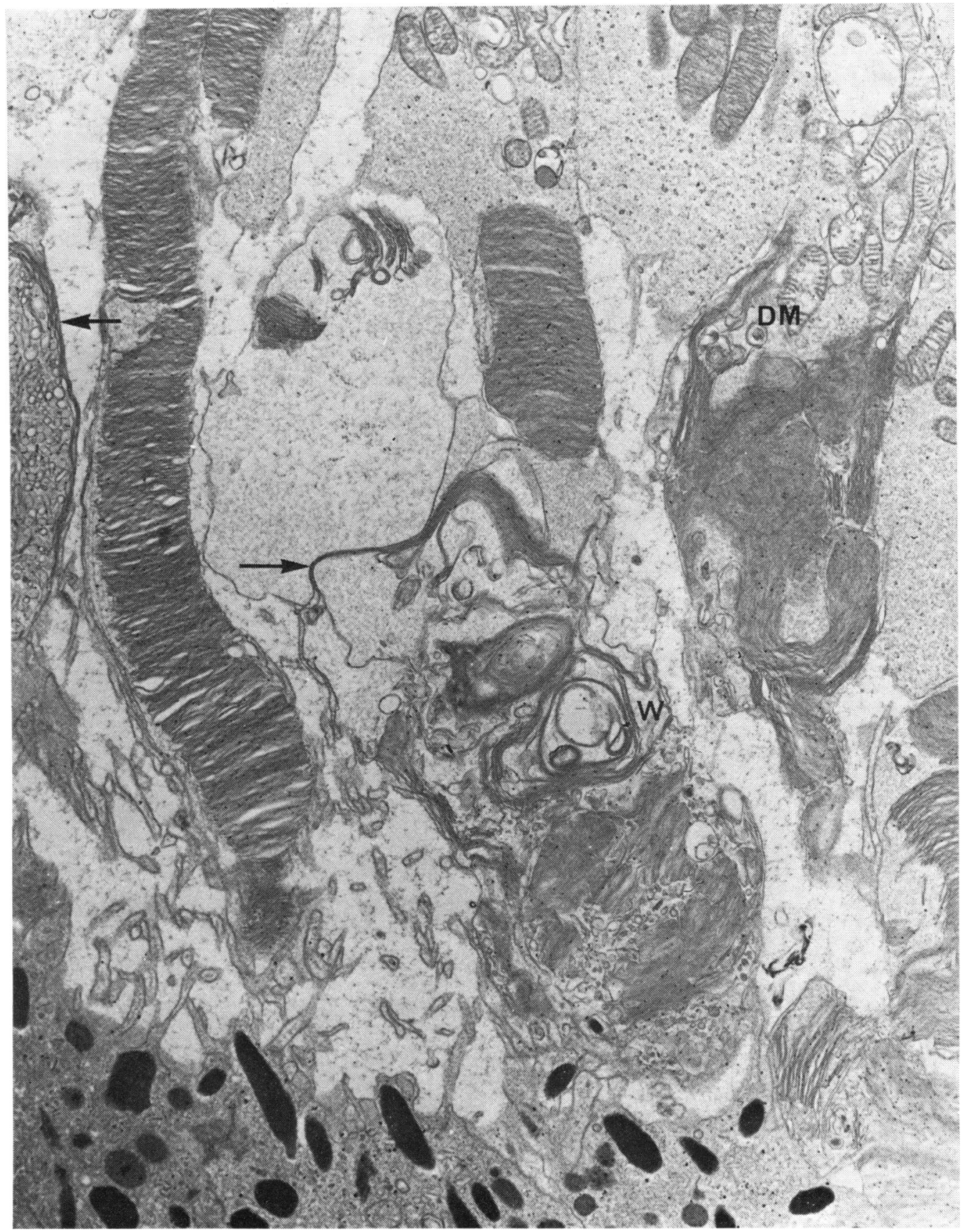

Fig. 10 Electron micrograph of nasal retina of eye removed 3 weeks after trauma, showing abnormal appearances of outer segments with whorls of plate material, $W$, surrounding cystic spaces, ribbon-like strands (arrows), and disc material, DM, being produced in the inner segments. $\times 11100$ 


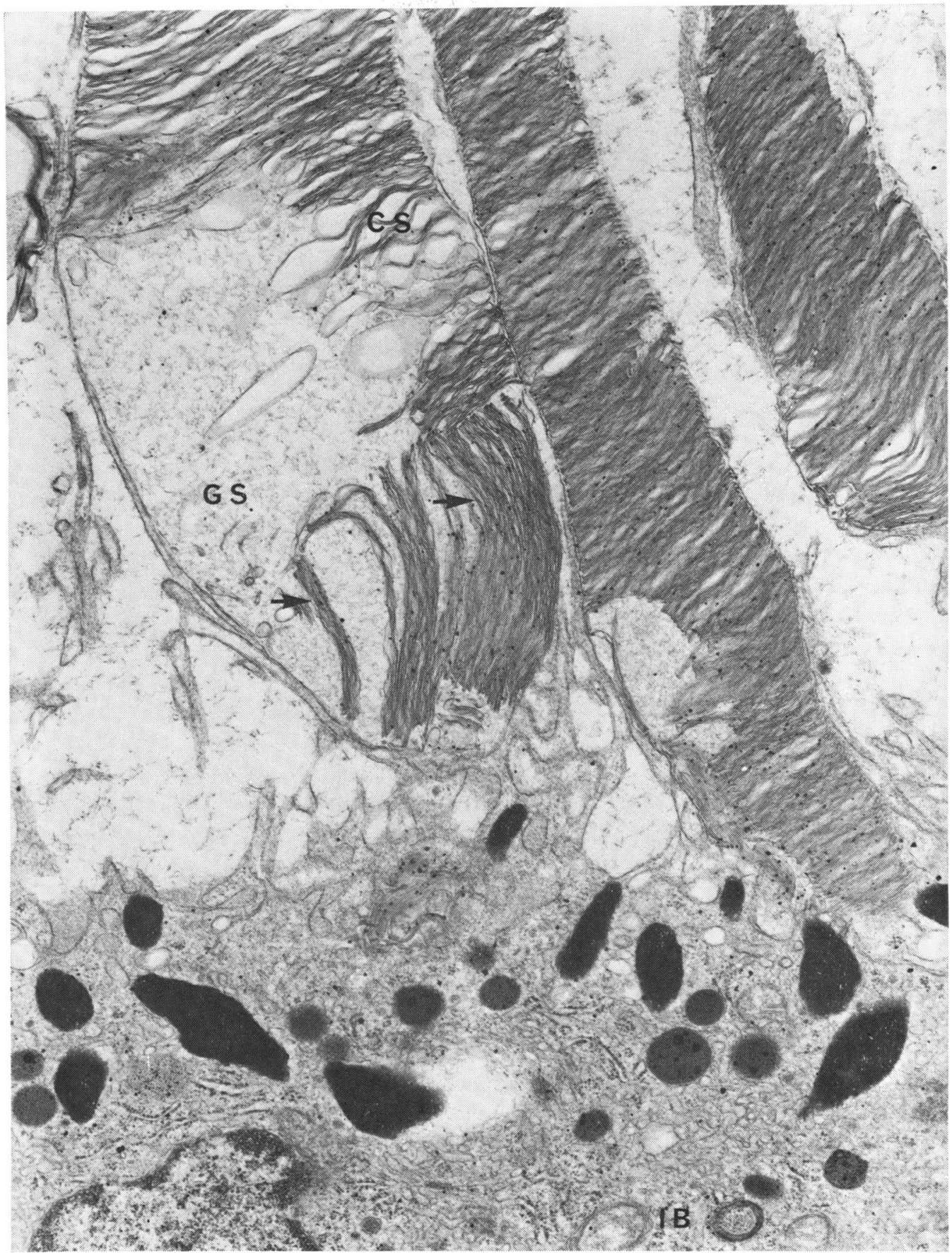

Fig. 11 Electron micrograph of nasal retina of eye removed 3 weeks after trauma, showing regenerating outer segments with cystic spaces, CS, between saccules, vertically orientated discs (arrows), and outer segments containing large quantities of amorphous ground substance, GS. Lamellar inclusion body, IB. $\times 18500$ 


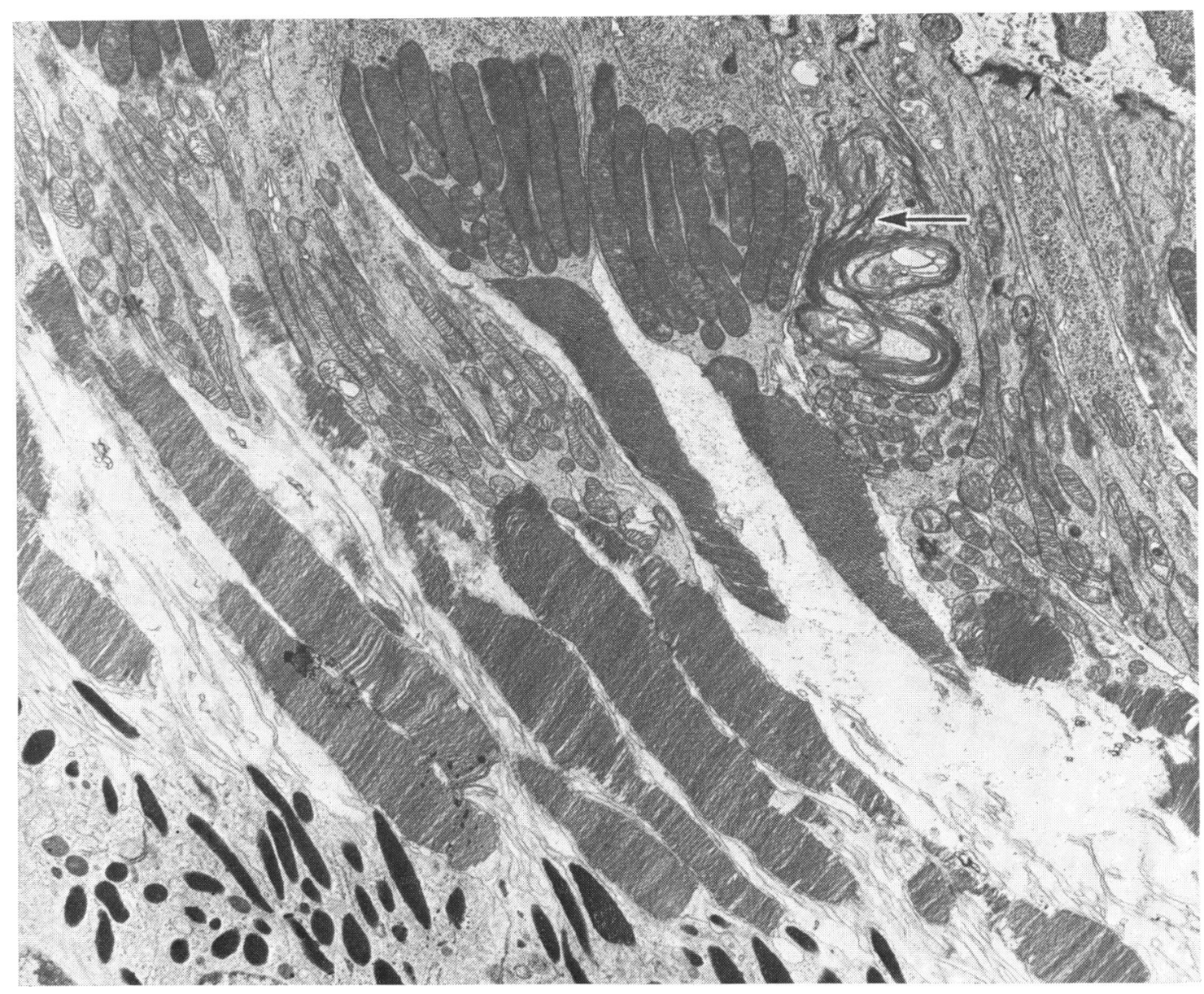

Fig. 12 Electron micrograph of nasal retina of eye removed 2 months after trauma. More normal looking photoreceptor outer segments. Some disordered regeneration of disc material is seen in the inner segment (arrow). $\times 6585$

limiting membrane after trauma. These structures may perhaps play some role in resisting distracting forces, thereby permitting shearing stresses to act only at certain levels of the retina.

Changes in the outer layers of the retina were maximal adjacent to the impact site and declined away from this point. No signs of contre-coup injuries were detected, thus supporting the in-vitro findings on pigs' eyes of Delori et al. (1969) that the forces generated by missiles of relatively high velocity and low mass impacting against the globe are transmitted via the ocular coats and not as block forces across the vitreous gel.

Repair of the pig's retinal pigment epithelium occurred quickly after trauma; the internal cell membrane was reconstituted and the apical processes reappeared within 7 days of injury. However, the dramatic disappearance of the fragmented outer segments within 1 week of trauma, with approximation of the inner segments of the photoreceptors to the retinal pigment epithelium, almost certainly did not come about as a result of phagocytosis by wandering macrophages, since very few of these cells were observed in our sections. It is possible that after trauma to the pig's retina enzymes released either from outer segments or from the damaged retinal pigment epithelium digested the disrupted outer segments in situ, a mechanism suggested by Cogan (1969) to explain similar changes which have been noted in man, and that the products of this process then rapidly diffused away into the surrounding tissues.

Regeneration of the outer segments, which was initially grossly disordered and characterised by the 
production of long streamers of lamellar material, incorrectly orientated plates, and whorl-like formations, some of which appeared to be formed in the inner segments, but becoming more orderly subsequently, has been reported after the reattachment of experimentally detached retinae (Kroll and Machemer, 1969). Since photoreceptor regeneration also occurs after blunt non-perforating mechanical injuries in pigs, it would appear that some elements of visual function may continue to improve over a period of weeks after blunt non perforating injuries in these animals.

The amount of pigment dispersion detected within the retina 2 months after trauma in pigs was minimal, both on ophthalmoscopic and on microscopical examination. It is possible that in this animal the retinal pigment epithelium shows a higher degree of stability to such injuries than in some other species, including man.

The degree of retinal damage which follows concussional injuries depends on the magnitude of the stresses imposed on this tissue. Traumatic retinal oedema, as described by Berlin (1873) is rapidly reversible, and Berlin stressed that retinal function quickly returns to a preinjury level. Experimental studies suggest that retinal opacification probably occurs in such cases because of the development of intracellular oedema at the level of the nerve fibre layer (Hart et al., 1975).

Fluorescein angiographic studies performed on patients presenting with Berlin's oedema showed no abnormal features, but in a patient who later developed retinal pigment epithelial disturbances visible on ophthalmoscopic examination fluorography performed soon after trauma revealed a temporary breakdown of the choroido-retinal barrier, with pooling of dye in the neural layers of the retina (Hart and Frank, 1975). In this case, with clear evidence of post-traumatic damage to the outer layers of the retina, central vision was initially severely impaired but continued to improve for several weeks, although no refractive error or loss of clarity of the optical media was present more than 4 days after trauma. The possibility exists that regeneration of disrupted photoreceptor outer segments noted in the retinae of pigs after concussive injuries may also, in favourable circumstances, occur in man.
We wish to thank Mr P. Darby for technical assistance and Mrs M. Roach for secretarial services.

This work was funded by the Medical Research Council, Grant No. 973/479/C.

\section{References}

Berlin, R. (1873). Zur Sogenannten Commotio Retinae. Klinische Monatsblätter für Augenheilkunde, 11, 42-78.

Bonnet, P., and Sedan, J. (1954). Sur le polymorphisme des éléments constituant le trouble de Berlin. Bulletin des Sociétés d'Ophtalmologie de France, 6, 591-597.

Cogan, D. G. (1969). Pseudo retinitis pigmentosa. Report of two cases of recent origin. Archives of Ophthalmology, 81, 45-53.

Crouch, E. R., and Apple, D. J. (1974). Post-traumatic migration of the retinal pigment epithelial melanin. American Journal of Ophthalmology, 78, 251-254.

Davidson, M. (1936). Minor sequelae of eye contusions. American Journal of Ophthalmology, 19, 757-769.

Delori, F., Pomerantzeff, O., and Cox, M. S. (1969). Deformation of the globe under high-speed impact: its relationship to contusion injuries. Investigative Ophthalmology, 8, 290-310.

Haab, O. (1888). Ueber bei Erkrankung der Macula Lutea. Report of the 7th International Ophthalmological Congress. Pp. 429-436. Bermann: Wiesbaden.

Hart, J. C. D., Blight, R., Cooper, R., and Papakostopoulos, D. (1975). Electrophysiological and pathological study of concussional injury: an experimental study. Transactions of the Ophthalmological Societies of the United Kingdom, 95, 326-334.

Hart, J. C. D., and Frank, H. J. (1975). Retinal opacification after blunt non-perforating concussional injuries to the globe: a clinical and retinal fluorescein angiographic study. Transactions of the Ophthalmological Societies of the United Kingdom, 95, 94-100.

Kroll, A. J. (1969). Secondary retinal detachment: Electron microscopy of retina and pigment epithelium. American Journal of Ophthalmology, 68, 223-237.

Kroll, A. J., and Machemer, R. (1968). Experimental retinal detachment in the owl monkey. III Electron microscopy of retina and pigment epithelium. American Journal of Ophthalmology, 66, 410-427.

Kroll, A. J., and Machemer, R. (1969). Experimental retinal detachment and reattachment in the Rhesus monkey. Electron microscopic comparison of rods and cones. American Journal of Ophthalmology, 68, 58-77.

Petropavlovskaya, G. A., and Bogoslovsky, A. I. (1966). Contusion of the eye ball. Clinico-physiological and experimental investigations. Vestnik of talmologii, 79, 34-40.

Rootman, J. (1971). Vascular system of the optic nerve head and retina of the pig. British Journal of Ophthalmology, 17, 808-819.

Tillema, A. (1937). Traumatic glaucoma. Archives of Ophthalmology, 17, 586-641.

Weidenthal, D. T., and Schepens, C. L. (1966). Peripheral fundus changes associated with ocular contusions. American Journal of Ophthalmology, 62, 465-477. 